\title{
Optical coherence tomography and fundus autofluorescence in children with infantile nystagmus syndrome and early-onset retinal dystrophy
}

\author{
Alma Kurent ${ }^{1}$ (D) Jelka Brecelj]², Branka Stirn-Kranjc ${ }^{2}$ (0) \\ 'Community Health Centre Ljubljana, Ljubljana, Slovenia \\ ${ }^{2}$ Eye Hospital, University Medical Centre Ljubljana, Ljubljana, Slovenia
}

\begin{abstract}
BACKGROUND: The purpose of the study was to compare morphological retinal differences in children with infantile nystagmus syndrome (INS) and early-onset retinal dystrophies (EORD) with healthy controls in order to evaluate the diagnostic value of optical coherence tomography (OCT) and fundus autofluorescence (FAF).

MATERIAL AND METHODS: Twenty-six children (mean age 10.0 years) were ophthalmologically and electrophysiologically diagnosed as INS and EORD. Their SD-OCT and FAF imaging was prospectively performed and compared with 36 controls (mean age 7.2 years).

RESULTS: Optical coherence tomography imaging was successfully completed in 17/26 (mean age 9.7 years) and FAF in 15/26 (mean age 11.1 years) children with EORD. OCT imaging was completed in 29/36 (mean age 8.9 years) and FAF in 15/19 (mean age 8.7 years) control children. In all six children with Leber congenital amaurosis (LCA) photoreceptor layer abnormalities were seen in the peripheral retina using SD-OCT, but macular images could not be obtained. In $5 / 5$ children with congenital stationary night blindness (CSNB) retinal thinning was found in all regions except the fovea. $2 / 6$ children with achromatopsia showed ellipsoid zone disruption with macular thinning. In 1/1 child with blue cone monochromatism reduced macular thickness was observed. In 5/5 children with cone-rod dystrophy the outer retinal signal intensity was decreased. In $2 / 3$ children with complete CSNB, slight foveal hypoautofluorescence was indicated. $1 / 4$ children with achromatopsia showed a central hyperautofluorescent ring. In 1/1 child with blue cone monochromatism slight foveal hypoautofluorescence was observed. 2/7 children with cone-rod dystrophy showed a central hyperautofluorescent ring, and additionally in 1/7 a macular hypoautofluorescence in one eye and an additional patch of hyperautofluorescence centrally in the other eye were found. CONCLUSION: The study demonstrated OCT and FAF as a non-invasive and fast diagnostic tool that defines morphological changes in early-onset retinal dystrophies in young children with nystagmus.
\end{abstract}

KEY WORDS: early-onset retinal dystrophy; optical coherence tomography; autofluorescence; children; nystagmus 


\section{INTRODUCTION}

Infantile nystagmus syndrome (INS) appears in the first few months of life and can be associated with early-onset retinal dystrophies (EORD). Among the most common EORD are Leber congenital amaurosis (LCA), congenital stationary night blindness (CSNB), achromatopsia, and cone-rod and rod-cone dystrophy [1, 2]. Fundus examination is often normal in early childhood, so early diagnosis of EORD is difficult. Electrophysiology as a standard diagnostic tool and later retinal morphology assessment with optical coherence tomography (OCT) and fundus autofluorescence (FAF) are suggested to aid in the diagnosis [3-6].

Both OCT and FAF are non-invasive diagnostic methods. In OCT, on the basis of the reconstruction of individual retinal images, a two- or multi-dimensional image of the retina and its layers is obtained and has become a standard diagnostic method in ophthalmology $[7,8]$. Rare OCT studies in the literature showed retinal changes in EORD, especially in children. Photoreceptor loss was described in LCA [9]. Preserved structure and retinal thinning, except in the fovea, was reported in CSNB [10]. A disruption or loss of the macular inner/outer segments junction of the photoreceptors was shown in achromatopsia $[3,11,12]$. Absence of an interdigitation zone was found in cone-rod dystrophy [13].

AF shows lipofuscin in the retinal pigment epithelium (RPE). In the case of incomplete decomposition photoreceptor membrane residues accumulate in RPE in the form of lipofuscin granules [14-16]. As in OCT, only a few studies have reported FAF characteristics in EORD. Normal FAF or parafoveal ring with mild to moderately increased autofluorescence was described in LCA [17]. Absence of an FAF signal in LCA patients with a mutation in RPE65 was reported $[6,18]$. No changes in FAF images were observed in CSNB [10]. Foveal and parafoveal hyperautofluorescence were detected in achromatopsia and in adult achromatopsia patients foveal hypoautofluorescence was described [4]. A hyperautofluorescence ring was noticed in some cases of cone-rod dystrophy [19].

Optical coherence tomography and FAF imaging in children with nystagmus is challenging due to unpredictable co-operation and unstable fixation in nystagmus $[6,20,21]$. The groups studied so far have usually been small and mostly included older children and adults using various OCT machines [9-13, 22-24]. Similarly, the retinal thickness val- ues of healthy children in the literature applied only to a group of children studied, while generally accepted normative values for the paediatric population are not yet available [25-29].

The purpose of this study was to morphologically assess the retina with OCT and FAF in 26 children with INS and EORD, and to compare the images with those from a group of 36 healthy children.

\section{MATERIAL AND METHODS}

In 26 children (mean age 10 years; 1 to 21 years) with INS and EORD, OCT and FAF imaging was performed. INS diagnosis was made clinically on the basis of bilateral and conjugate ocular movements with no change of direction in different gaze directions, and in all children the onset of nystagmus was observed within the first six months after birth. EORD was diagnosed clinically and electrophysiologically as: LCA in six children, CSNB in five children, achromatopsia in six children, blue cone monochromatism in one child, cone-rod dystrophy without known systemic disease in four children, and cone-rod dystrophy with genetic systemic disease in three children (the first child with heart problems and obesity, the second child with nephronophthisis, and the third child with thiamine-responsive megaloblastic anaemia syndrome), and rod-cone dystrophy with chondrodysplasia and neurologic disorder in one child.

Ophthalmologic examinations and ERG recordings of these children were presented in previous studies $[31,32]$. This study was performed with the informed consent of the parents of all of the children who participated, and it was approved by the Slovene Medical Ethics Committee (No. 30/02/12).

Optical coherence tomography images were obtained in mydriasis with an OCT Topcon 3D OCT-1000 (Topcon Medical Systems, Inc., Topcon Corporation of Tokyo, Japan) and/or Spectralis HRA + OCT (Heidelberg Engineering, Inc., Heidelberg, Germany). The retinal thickness and total macular volume were determined using the OCT apparatus software. Retinal thickness was determined in the Early Treatment Diabetic Retinopathy Study (ETDRS) mode with circular diameters of 1,3 , and $6 \mathrm{~mm}$. The thickness of the retina was measured in nine regions of the macula: the fovea, the inner lower, the outer lower, the inner nasal, the outer nasal, the inner upper, the outer upper, the inner temporal, and the outer temporal regions, 
similarly to other studies [26-30]. The OCT retinal thickness and total macular volume values in the children with EORD were compared statistically with the values of healthy children using SPSS software (IBM SPSS Statistics, Armonk, New York, USA), with differences significant if $\mathrm{p}<0.05$.

AF images were obtained in mydriasis with a fundus camera Topcon TRC-50IX (Topcon Medical Systems, Inc., Topcon Corporation of Tokyo, Japan) and/or Spectralis HRA + OCT (Heidelberg Engineering, Inc., Heidelberg, Germany).

\section{RESULTS}

Optical coherence tomography images were successfully obtained in at least one scan in 17 (65.4\%) children (mean age 9.7 years; 1 to 21 years) and FAF images in 15 (57.7\%) children with EORD (mean age 11.1 years; 4 to 21 years). Macular thickness and total macular volume were analysed in OCT images in 13 (50\%) children (mean age 10.8 years; 2.5 to 21 years). Older children were included in the study during their follow-up but were diagnosed earlier. Retinal thickness values and total macular volume are shown in Table I.

Thirty-six healthy children (mean age 7.2 years; 4 months to 18 years) had OCT imaging. OCT images were successfully obtained in $29(80.6 \%)$ children (mean age 8.9 years; 2 to 18 years), retinal thickness values and total macular volume are shown in Table 1. Nineteen healthy children (mean age 7.7 years; 6 months to 18 years) had FAF imaging. Fundus autofluorescence images were successfully obtained in 15 (79\%) children (mean age 8.7 years; 8 months to 18 years).

In children with LCA photoreceptor layer abnormalities were seen in the peripheral retina in SD-OCT (Fig. 1). In the six children with LCA, OCT and FAF macular images were not successfully obtained, due to rowing eye movements and lack of fixation.

Optical coherence tomography macular images were successfully obtained in all five children with CSNB (three with complete and two with incomplete type). OCT images in CSNB showed macular thickness reduction in all macular regions except the fovea (Fig. 1) and total macular volume reduction (Tab. I). OCT macular images in six children with achromatopsia revealed no structural abnormalities in four children, while in two children ellipsoid zone disruption was evident (Fig. 1). Reduced macular thickness was indicated, but the

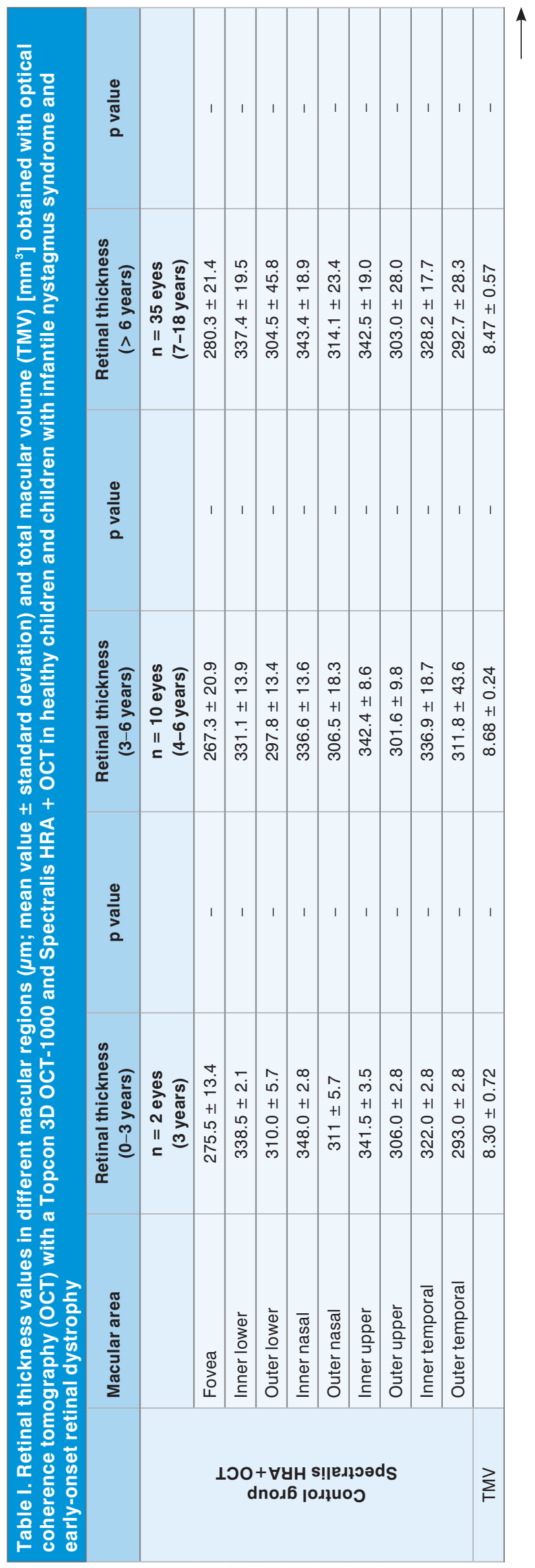




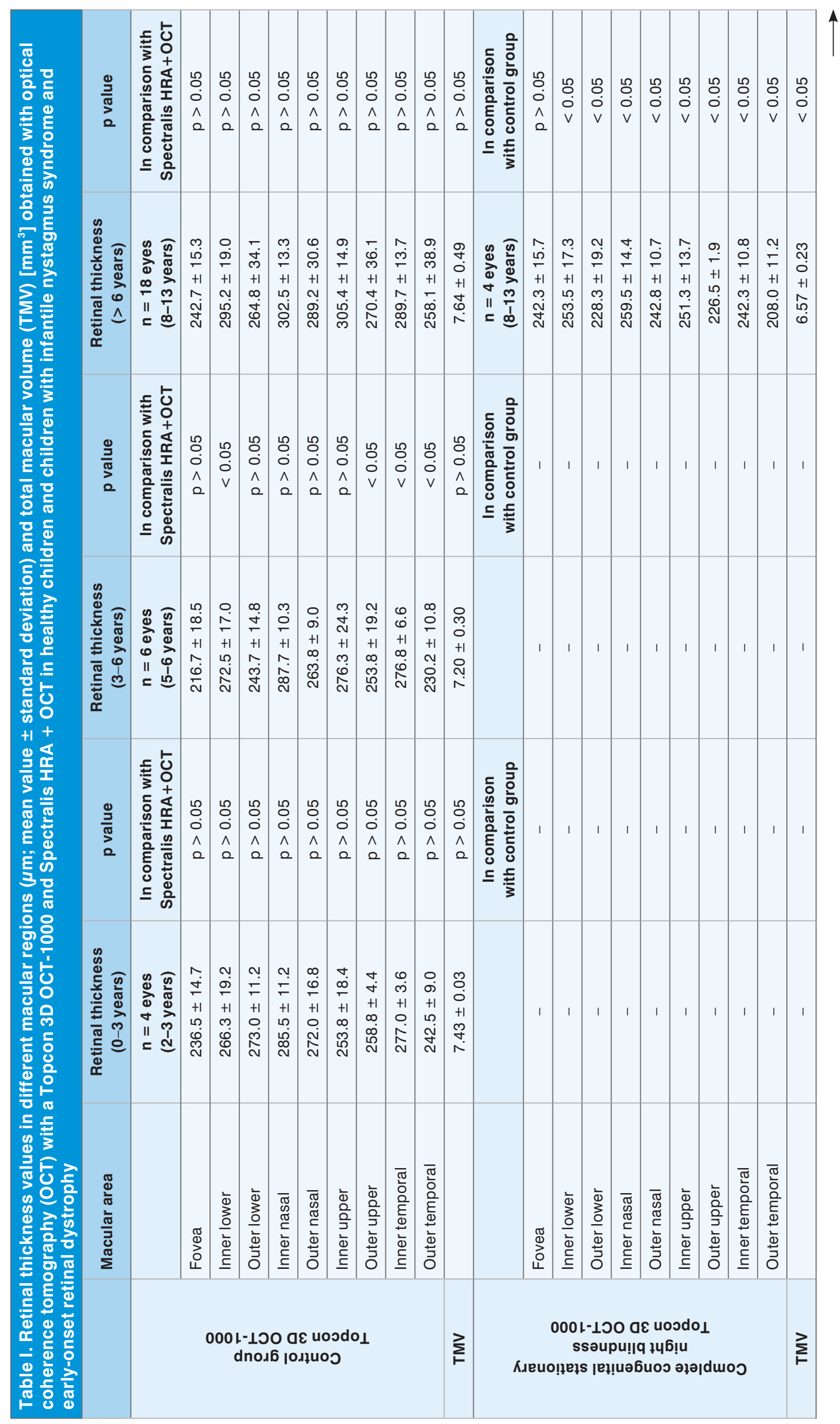




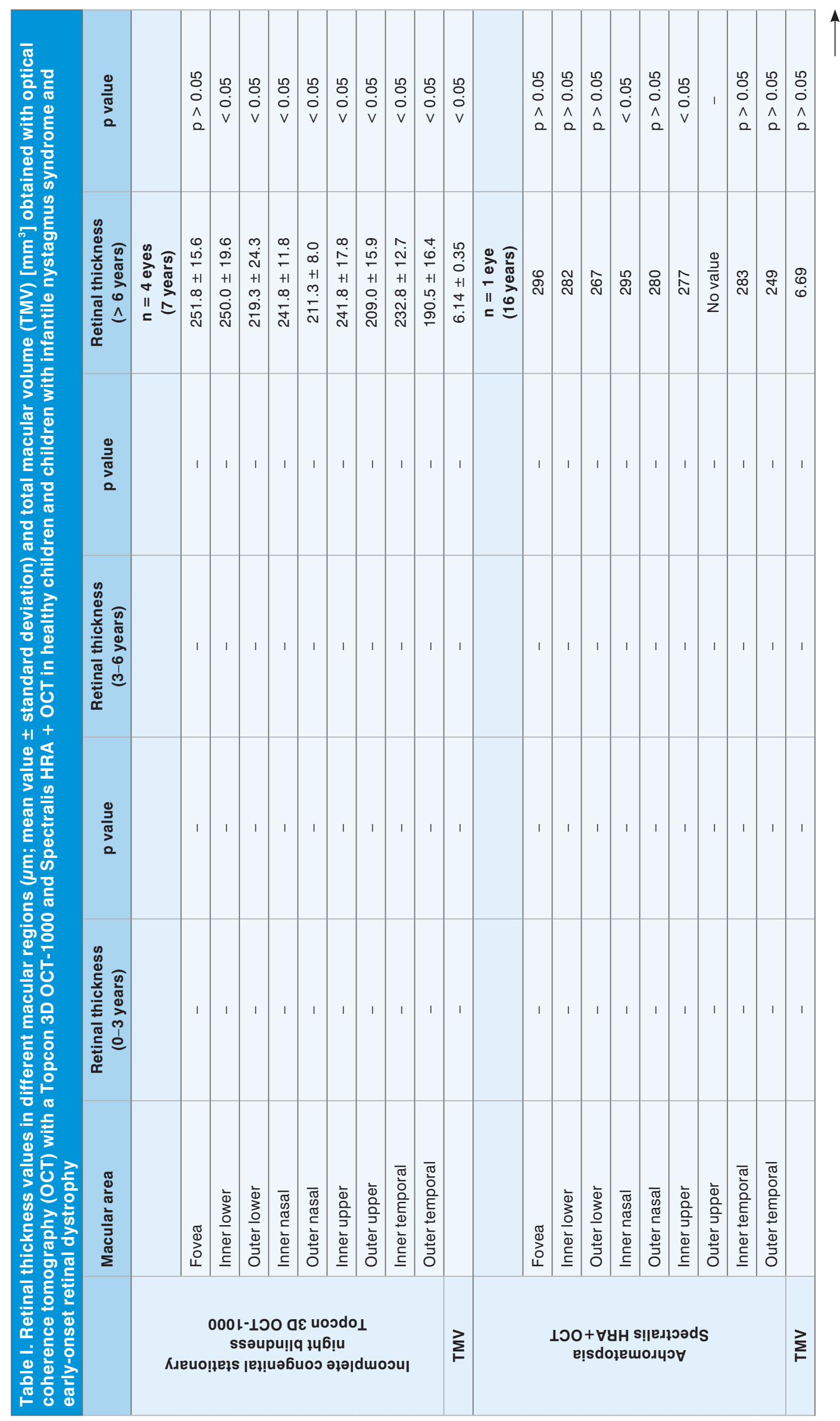




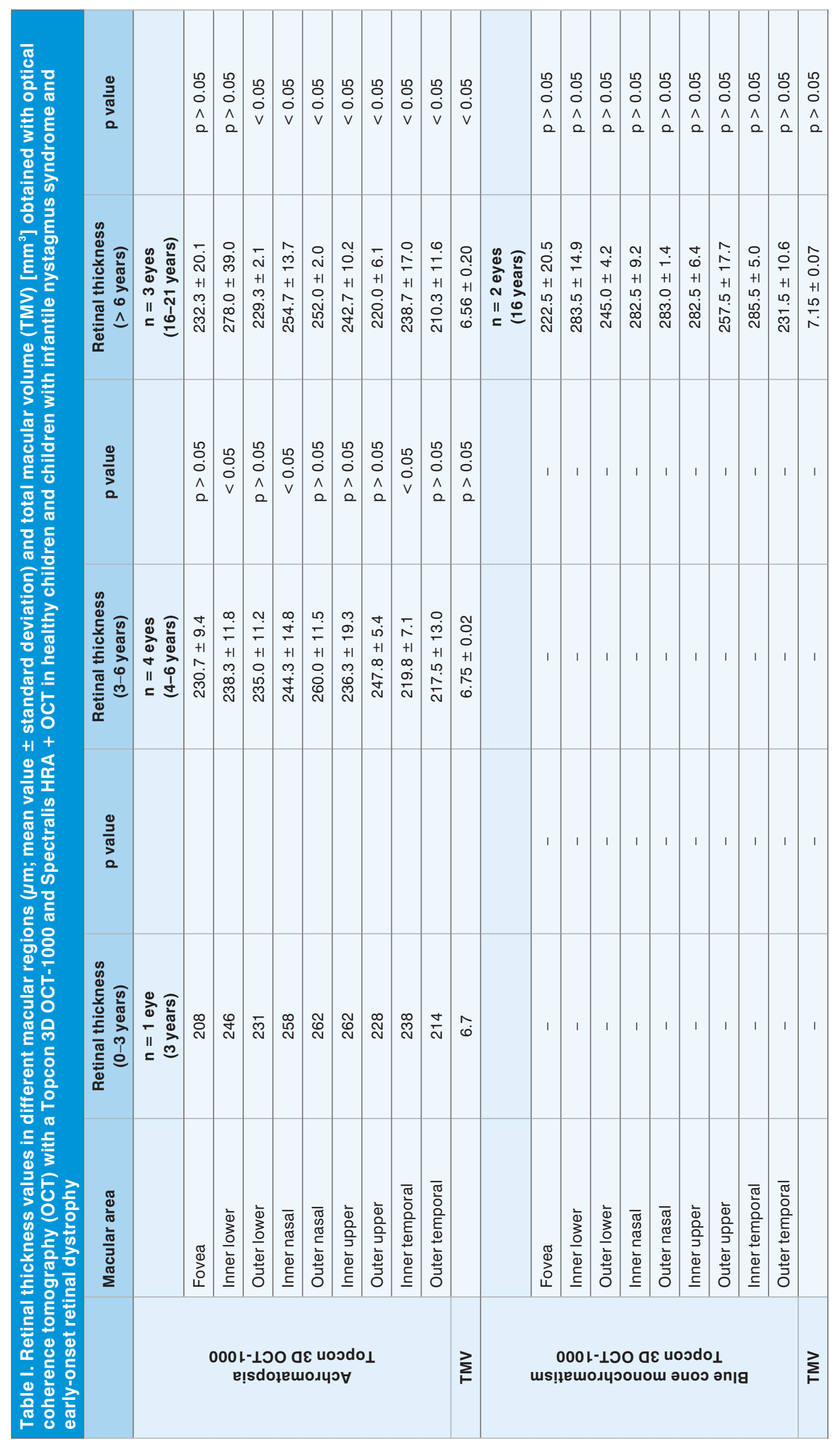




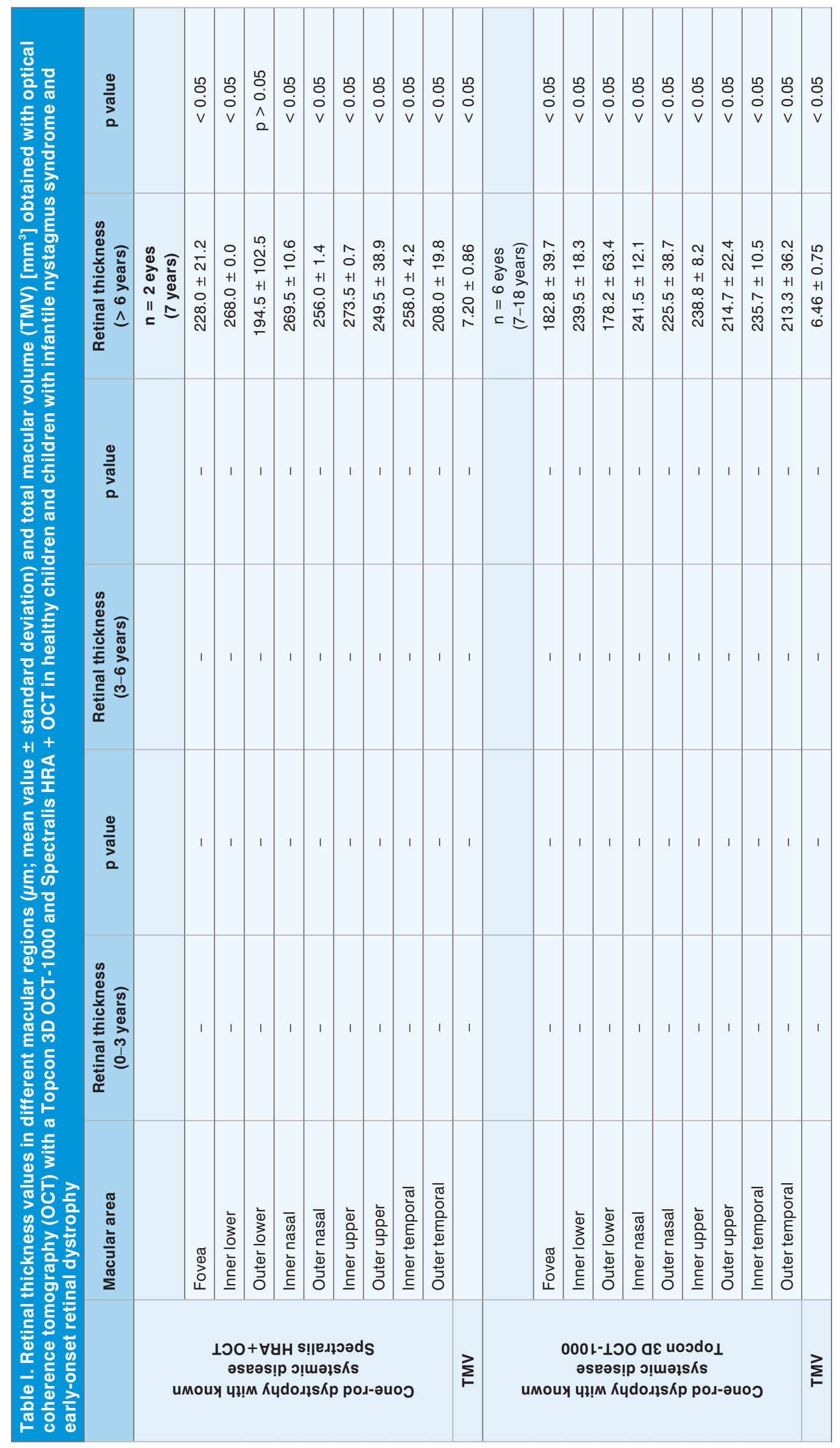



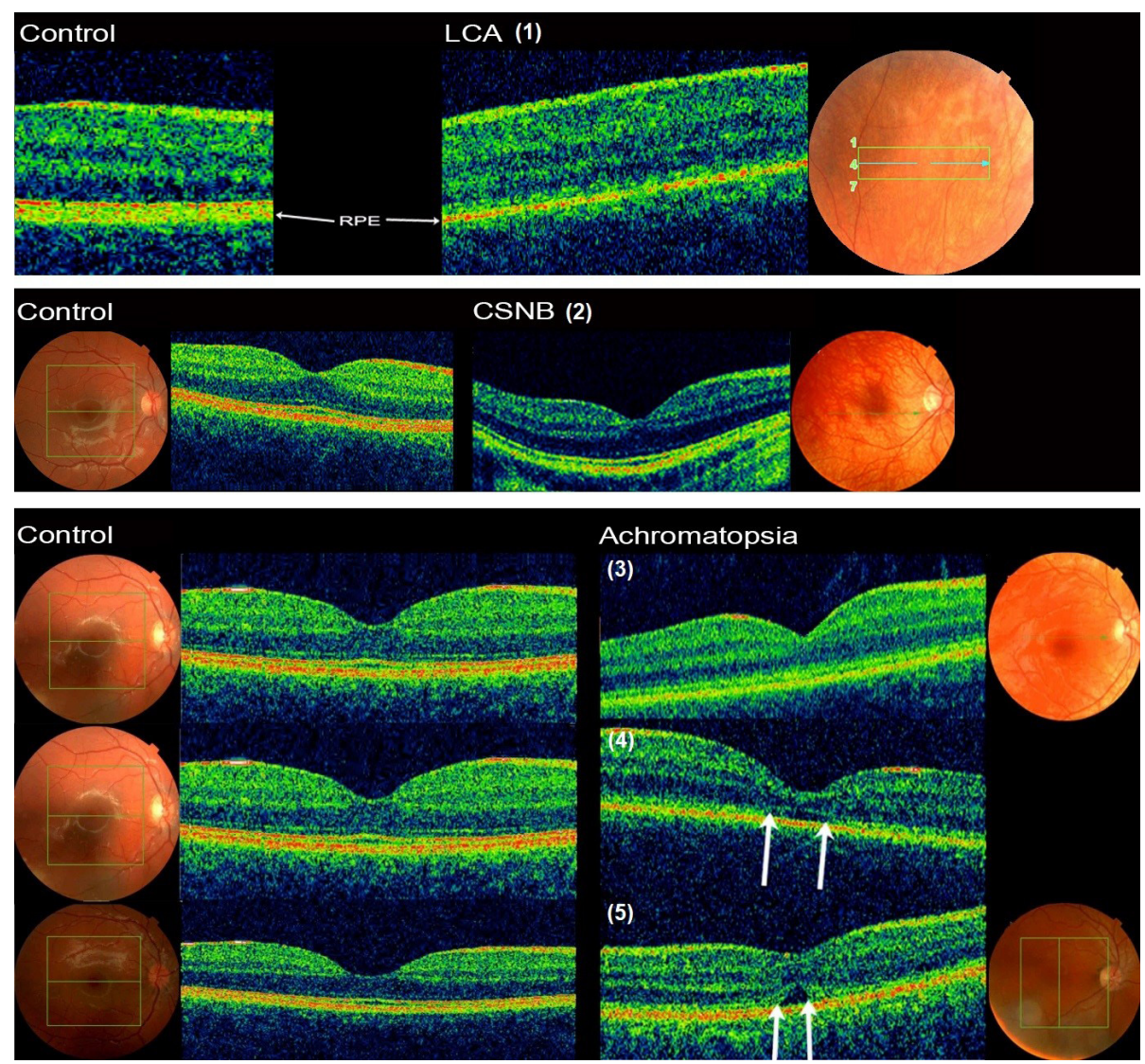

FIGURE 1. Optical coherence tomography (OCT) images (Topcon 3D 1000): (1) in a 4-year-old child with Leber congenital amaurosis (LCA) showing photoreceptor layer abnormalities in the peripheral retina compared to a healthy 4-year-old child (RPE, retinal pigment epithelium); (2) in a 6-year-old child with complete congenital stationary night blindness (CSNB) showing thinning of the retina without other structural abnormalities compared to a healthy 7-year old child; (3) in a 2.5-year-old child with achromatopsia showing no visible structural abnormalities compared to the image of healthy 3-year-old child; $(4)$ in a 2-year-old boy with achromatopsia showing visible ellipsoid zone disruption (between arrows) compared to a healthy 3-year-old child (fundus image was of poor quality due to the nystagmus); (5) in a 21-year-old child with achromatopsia showing visible ellipsoid zone disruption (between arrows) compared to a healthy 12-year-old child

difference was not statistically significant in all regions (Tab. I). In $1 / 1$ child with blue cone monochromatism no OCT structural abnormalities were noticed (Fig. 2). Macular thickness and total macular volume reductions were not statistically significant (Tab. I). OCT macular images were successfully obtained in 5/7 children with cone-rod dystrophy. In two children with cone-rod dystrophy without known systemic disease and in three children with known systemic disease OCT images showed reduced intensity of the outer retinal signal (Fig. 2), macular thinning, and total macular volume reduction (Tab. I). In the group of children with cone-rod dystrophy without known systemic disease, the obtained images were not of sufficient quality for quantitative analysis.

Fundus autofluorescence images were obtained in $2 / 3$ children with complete CSNB, showing slight hypoautofluorescence (Fig. 3). In four children with achromatopsia, FAF showed no abnormalities in three children, and in one child a central hyperautofluorescent ring was found (Fig. 4). Fundus autofluorescence images in a child with blue cone monochromatism indicated slight hypoautofluorescence in the fovea (Fig. 3). Fundus autofluorescence images were successfully obtained in seven children with cone-rod dystrophy, showing no abnormalities in all four children without systemic disease, while in all three children with cone-rod dystrophy with known systemic disease the following changes in FAF were observed: in two children (in one child - heart disease and obesity, and in another child - nephronophthisis) the hyperautofluorescent ring was centrally located, and in one child with thiamine-responsive megaloblastic anaemia syndrome macular hypoautofluorescence in the right eye was seen, while in the left eye additional 

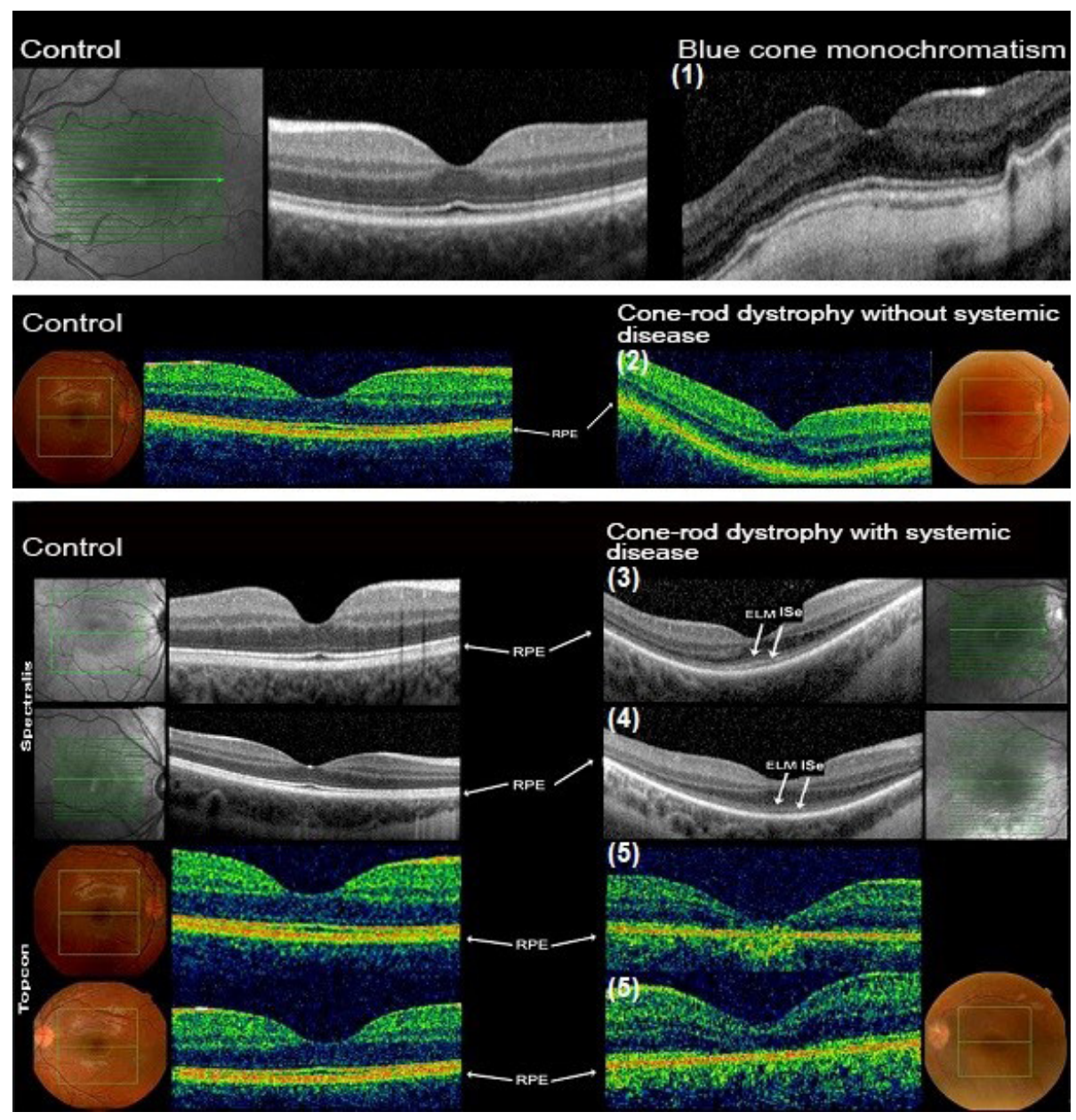

FIGURE 2. Optical coherence tomography (OCT) images: (1) in a 18-year-old child with blue cone monochromatism showing no visible structural abnormalities except the thinning of the retina compared to a healthy 15 -year-old child (fundus image was of poor quality due to the nystagmus) (Spectralis); (2) in a 17-year-old cone-rod dystrophy child without known systemic disease indicating a reduced signal in the outer retina compared to the 12 year-old healthy child (Topcon); (3) in a 13-year-old child with cone-rod dystrophy with cardiac failure and obesity showing reduced signal in the outer retina compared to a 12-year-old child (Spectralis); (4) in a 7-year-old with conerod dystrophy and nephronophthisis showing reduced signal in the outer retina compared to a 7-year-old healthy child (Spectralis); (5) of the right and the left eye of a 15-year-old cone-rod dystrophy patient with thiamine-dependent megaloblastic anaemia syndrome showing reduced signal in the outer retina compared to a 12-year-old healthy child (Topcon). In the Spectralis HRA + OCT, a reduced signal of the inner segment ellipsoid and an external limiting membrane is seen more clearly than in the Topcon 3D OCT-1000. ELM — external limiting membrane; ISe — inner segment ellipsoid; RPE — retinal pigment epithelium

hyperautofluorescent patch was found (Fig. 4). No abnormalities were noticed in FAF images in a child with rod-cone dystrophy with chondrodysplasia and neurologic disorder (Fig. 3).

\section{DISCUSSION}

In this study we observed significant OCT and FAF changes in comparison with healthy controls in children with INS and EORD. The morphological changes: retinal thinning in CSNB in all regions, except fovea, ellipsoid zone disruption and central hyperautofluorescent ring in achromatopsia, and decreased outer retinal signal intensity with central hyperautofluorescent ring in cone-rod dystrophy were comparable to the literature $[3,4$, $10,13,19]$. Also, data on the morphological retinal appearance in healthy children was important in the study for comparison with children with INS and EORD, and it complements the existing literature $[25,26,28-30,33]$. The abnormal photoreceptor layer of the peripheral retina found in the present study in LCA patients was also reported in fovea in the study in nine patients, aged 6 to 17 years, with LCA and the mutation in RPE65 [9]. Wabbels et al. reported difficulties in performing FAF imaging in LCA patients [6], similarly as in the present study. 


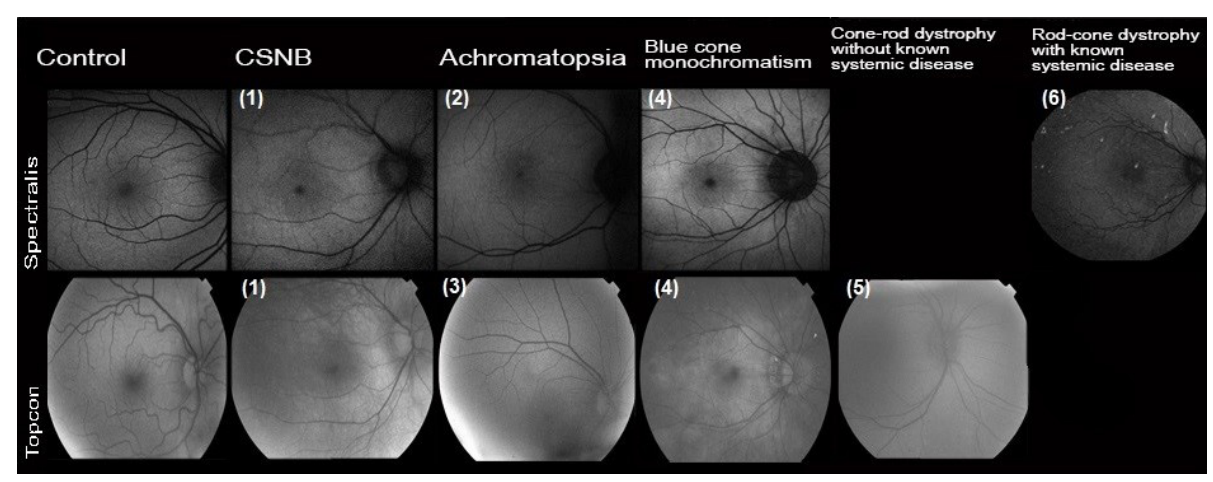

FIGURE 3. Autofluorescence (AF) images: (1) of a 7-year-old child with complete congenital stationary night blindness (CSNB) (the same patient as in Figure 1) indicating slight foveal hypoautofluorescence; (2) of a 19-year-old patient with achromatopsia with no visible abnormalities; (3) of a 6 -year-old patient with achromatopsia with no visible abnormalities; (4) of an 18-year-old patient with blue cone monochromatism (the same patient as in Figure 2) indicating slight foveal hypoautofluorescence; (5) of a 17-year-old patient with cone-rod dystrophy without known systemic disease (the same patient as in Figure 2) with no visible abnormalities; (6) and in a 14-year-old patient with rod-cone dystrophy with known systemic disease with no visible abnormalities. Controls were 5.5- (Spectralis) and 7-year-old (Topcon) healthy children.

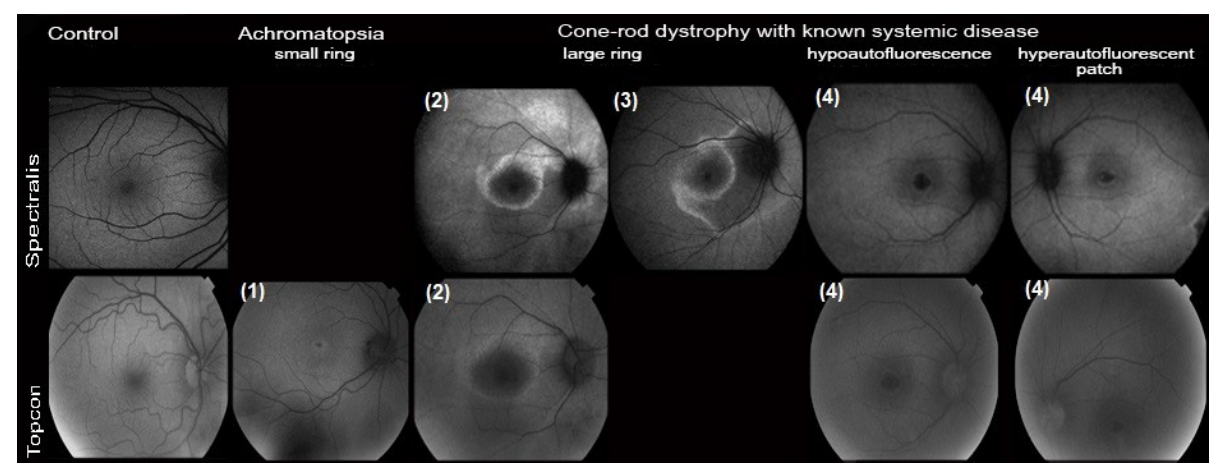

FIGURE 4. Autofluorescence (AF) images: (1) of a 21-year-old patient with achromatopsia (the same patient as in Figure 1) with a small hyperautofluorescent ring centrally; (2) of a 7-year-old child with cone-rod dystrophy with known systemic disease patients (the same patient as in Figure 2) with a large hyperautofluorescent ring centrally; (3) of a 14-year-old child with cone-rod dystrophy with known systemic disease patients (the same patient as in Figure 2) with a large hyperautofluorescent ring centrally; (4) of both eyes of a 15-year-old patient with thiamine-dependent megaloblastic anaemia syndrome (the same patient as in Figure 2). On the FAF image of the right eye, hypoautofluorescence is visible, and on the FAF image of the left eye an additional hyperautofluorescent patch in the macula is visible. Controls were 5.5- (Spectralis) and 7-year-old (Topcon) healthy children

Chen et al. [10] observed qualitatively normal OCT results and a statistically significant macular thinning outside the fovea in five incomplete type CSNB patients aged 6, 9, 11, 12, and 69 years, compared to four myopic controls. Similar changes were found in the present study. Slight foveal hypoautofluorescence in two children with complete CSNB in FAF images was indicated in the present study. CSNB patients in the comparable study demonstrated qualitatively normal FAF images [10].

Ellipsoid zone disruption in two children with achromatopsia in the present study was also reported in the literature, both in adults [11] and in children [24]. In the study from Genead et al. [11], normal ellipsoid zone was found in OCT in two children (13 and 17 years old) with achromatopsia, which was similar to the four children in the present study. The lower macular thickness values and reduced total macular volume in achromatopsia patients in the present study are comparable to the study from Varsányi et al. [22], where macular thinning except in fovea and reduced total macular volume were found in eight patients with achromatopsia (aged 4 to 67 years).

A comparable study (4) of $4 / 10$ children with achromatopsia showed foveal and parafoveal hyperautofluorescence ( 7 months to 14 years old). Similarly, a patient with achromatopsia in the present study, followed until 21 years of age, showed a hyperautofluorescent ring [Fig. 4; (1)] in addition to 
the ellipsoid zone disruption seen on OCT [Fig. 1; (5)]. Changes found on OCT and FAF in the same child were helpful in the diagnostic process, which makes OCT and FAF a valuable diagnostic tool especially in children without gross fundus abnormalities and in younger children with EORD. In the study (4), normal FAF in one 11-year-old child with achromatopsia was found, which is similar to the three children, aged 4 to 19 years, from the present study.

Barthelmes et al. [23] observed a significantly reduced foveal thickness and missing external limiting membrane in six patients with a blue cone monochromatism (average age 33.2 years). In the 18-year-old blue cone monochromatism patient from the present study, there were no structural abnormalities. In the study, a 54-year-old patient with blue cone monochromatism showed increased autofluorescence on the edges of atrophic lesion in the macula due to the accumulation of lipofuscin, which was a sign of a progressive dystrophic process [34]. Follow-up imaging in the future may show additional changes in an 18-year-old blue cone monochromatism patient from the present study.

The reduced signal in the photoreceptor layer and external limiting membrane (ELM) in patients with cone-rod dystrophy without known systemic disease from the present study was comparable to the findings from Lima et al. [13]. In their study, 12 subjects with cone-rod dystrophy (average age 30 years) showed the absence of a layer between the cone outer segments and apical processes of the RPE, the intensity of the ellipsoid of the inner segments of the photoreceptors was decreased, and in most patients ELM was absent in the foveal region [13].

The hyperautofluorescent ring was reported in the literature in some of the 41 adults with cone-rod dystrophy [19]. Similarly, it was found in two patients with cone-rod dystrophy with known systemic disease in the present study [Fig. 4; (2), (3)] in addition to reduced outer retinal signal intensity on OCT [Fig. 2; (3), (4)]. A central area of reduced FAF surrounded by small disseminated spots of increased FAF was reported in two children with cone-rod dystrophy (6). A patient with cone-rod dystrophy and thiamine-responsive megaloblastic anaemia syndrome in the present study showed a difference in the signal intensity in FAF from the right and the left eye [Fig. 4; (4)], in addition to reduced outer retinal signal intensity seen on OCT [Fig. 2; (5)], which might indicate a different stage of the disease process. A hyperautofluorescent ring was observed in a study in 12 children with rod-cone dystrophy (2 to 15 years old) (6), while in the present study in a 14-year-old patient with rod-cone dystrophy and chondrodysplasia and neurologic disorder no abnormalities were found in AF.

In the present study, which included only paediatric patients, OCT imaging was successful in $65.4 \%$. According to the literature, OCT imaging was successful in more than $95 \%$ (age range 2 to 68 years) of patients with nystagmus [20,35]. The youngest children with a nystagmus and successful OCT imaging were two years [35] and six years old [20]. Similarly, in the present study OCT imaging was generally successful in children over six years of age, but it was also successful in five children from two years of age. On the other hand, studies in the literature $[21,36,37]$ report good feasibility of handheld OCT in young children (mean age 3.5 and 3.7 years) with nystagmus. In addition to age, the success of OCT imaging also depended on the specific EORD characteristics, e.g. in children with LCA, nystagmus was very pronounced, and therefore imaging was less successful than in other dystrophies. In the literature [6] it was reported that in children with congenital retinal disease, some of whom had a pronounced nystagmus, FAF imaging was successful in children, mostly older than five years. Similarly, most of the children in the present study were older than six years.

The proportion of healthy children in which OCT images were successfully obtained in the study was similar to the literature and was above $80 \%$ [30]. The youngest healthy child in the study, in whom OCT imaging was successful, was two years old, while in comparable studies, children were older than three years of age [25-28]. In our experience, in addition to age, also good cooperation and motivation of the child contributed to successfully obtaining OCT images. The youngest healthy child in the study, in whom FAF imaging was successful, was eight months old, while in comparable studies children were over four years of age $[6,38]$.

Two different OCT and FAF machines were used in the study. The differences in retinal thickness between different OCTs are influenced by several factors, including different analysis algorithms that determine the internal and external retinal limits and the different image quality [39]. Fundus autofluorescence changes in the Topcon TRC-50IX images were less pronounced than those in the Spectralis HRA + OCT. This is probably mostly due 
to different imaging techniques [16]. A limitation of the present study is that some of the statistical comparisons with the normative values are less reliable due to the small numbers of children in some groups.

\section{CONCLUSION}

Assessing retinal morphology in children with INS and EORD with OCT and FAF is a less frequently reported method of studying the disease processes in these patients. The morphological characteristics identified in this study suggested diagnostic value of OCT and FAF in children with INS and EORD. The study demonstrated that OCT and FAF imaging can be successfully performed also in children with nystagmus and used as a non-invasive and fast diagnostic tool for defining morphological changes in early onset retinal dystrophies with the possibility to follow-up disease progression.

\section{ACKNOWLEDGEMENTS}

The authors thank Darko Perovšek, Barbara Klemenc and Mitja Pipan for their assistance with the OCT and FAF imaging. The abstract of the study was partially presented at $9^{\text {th }}$ Slovenian Congress of Ophthalmology, 28-30 June 2012, Portorož, Slovenia and SOE 2019 Congress, 13-16 June 2019, Nice, France. The study was financially supported by the Slovenian Research Agency, Grant No. P3-0333.

\section{DECLARATION OF COMPETING/CONFLICTS OF INTEREST}

The authors do not have any conflicts of interest.

\section{REFERENCES}

1. Casteels I, Harris CM, Shawkat $\mathrm{F}$, et al. Nystagmus in infancy. $\mathrm{Br} \mathrm{J}$ Ophthalmol. 1992; 76(7): 434-437, doi: 10.1136/bjo.76.7.434, indexed in Pubmed: 1627515.

2. CEMAS Working Group. National Eye Institute Sponsored Workshop and Publication on The Classification of Eye Movement Abnormalities and Strabismus (CEMAS). National Institutes of Health, The National Eye Institute Publications (www.nei.nih.gov). 2001.

3. Thomas MG, Gottlob I. Optical coherence tomography studies provides new insights into diagnosis and prognosis of infantile nystagmus: a review. Strabismus. 2012; 20(4): 175-180, doi: 10.3109/09273972. 2012.735336, indexed in Pubmed: 23211143.

4. Fahim AT, Khan NW, Zahid S, et al. Diagnostic fundus autofluorescence patterns in achromatopsia. Am J Ophthalmol. 2013; 156(6): 1211-1219.e2, doi: 10.1016/j.ajo.2013.06.033, indexed in Pubmed: 23972307.

5. Good PA, Searle AE, Campbell S, et al. Value of the ERG in congenital nystagmus. Br J Ophthalmol. 1989; 73(7): 512-515, doi: 10.1136/ bjo.73.7.512, indexed in Pubmed: 2788016

6. Wabbels B, Demmler A, Paunescu K, et al. Fundus autofluorescence in children and teenagers with hereditary retinal diseases. Graefes Arch
Clin Exp Ophthalmol. 2006; 244(1): 36-45, doi: 10.1007/s00417-0050043-2, indexed in Pubmed: 16034607.

7. Huang $D$, Swanson $E A$, Lin $C P$, et al. Optical coherence tomography. Science. 1991; 254(5035): 1178-1181, doi: 10.1126/science.1957169, indexed in Pubmed: 1957169.

8. Helb HM, Charbel Issa P, Fleckenstein M, et al. Clinical evaluation of simultaneous confocal scanning laser ophthalmoscopy imaging combined with high-resolution, spectral-domain optical coherence tomography. Acta Ophthalmol. 2010; 88(8): 842-849, doi: 10.1111/j. 1755-3768.2009.01602.x, indexed in Pubmed: 19706019.

9. Jacobson SG, Cideciyan AV, Aleman TS, et al. Photoreceptor layer topography in children with leber congenital amaurosis caused by RPE65 mutations. Invest Ophthalmol Vis Sci. 2008; 49(10): 4573-4577, doi: 10.1167/iovs.08-2121, indexed in Pubmed: 18539930.

10. Chen RWS, Greenberg JP, Lazow MA, et al. Autofluorescence imaging and spectral-domain optical coherence tomography in incomplete congenital stationary night blindness and comparison with retinitis pigmentosa. Am J Ophthalmol. 2012; 153(1): 143-54.e2, doi: 10.1016/j. ajo.2011.06.018, indexed in Pubmed: 21920492.

11. Genead MA, Fishman GA, Rha J, et al. Photoreceptor structure and function in patients with congenital achromatopsia. Invest Ophthalmol Vis Sci. 2011; 52(10): 7298-7308, doi: 10.1167/iovs.11-7762, indexed in Pubmed: 21778272.

12. Hood DC, Zhang $X$, Ramachandran R, et al. The inner segment/outer segment border seen on optical coherence tomography is less intense in patients with diminished cone function. Invest Ophthalmol Vis Sci. 2011; 52(13): 9703-9709, doi: 10.1167/iovs.11-8650, indexed in Pubmed: 22110066.

13. Lima LH, Sallum JMF, Spaide RF. Outer retina analysis by optical coherence tomography in cone-rod dystrophy patients. Retina. 2013; 33(9): 1877-1880, doi: 10.1097/IAE.0b013e31829234e6, indexed in Pubmed: 23648999.

14. von Rückmann A, Fitzke FW, Bird AC. Distribution of fundus autofluorescence with a scanning laser ophthalmoscope. Br J Ophthalmol. 1995; 79(5): 407-412, doi: 10.1136/bjo.79.5.407, indexed in Pubmed: 7612549 .

15. Lois N, Halfyard AS, Bird AC, et al. Quantitative evaluation of fundus autofluorescence imaged nin vivo« in eyes with retinal disease. $\mathrm{Br} \mathrm{J}$ Ophthalmol. 2000; 84(7): 741-745, doi: 10.1136/bjo.84.7.741, indexed in Pubmed: 10873986.

16. Schmitz-Valckenberg S, Holz FG, Bird AC, et al. Fundus Autofluorescence Imaging. Review and Perspectives. Retina. 2008; 28(3): 385-409, doi: 10.1097/IAE.0b013e318164a907, indexed in Pubmed: 18327131.

17. Scholl HPN, Chong NH, Robson AG, et al. Fundus autofluorescence in patients with leber congenital amaurosis. Invest Ophthalmol Vis Sci. 2004; 45(8): 2747-2752, doi: 10.1167/iovs.03-1208, indexed in Pubmed: 15277500.

18. Lorenz $B$, Wabbels $B$, Wegscheider $E$, et al. Lack of fundus autofluorescence to 488 nanometers from childhood on in patients with early-onset severe retinal dystrophy associated with mutations in RPE65. Ophthalmology. 2004; 111(8): 1585-1594, doi: 10.1016/j. ophtha.2004.01.033, indexed in Pubmed: 15288992.

19. Oishi M, Oishi A, Ogino $K$, et al. Wide-field fundus autofluorescence abnormalities and visual function in patients with cone and cone-rod dystrophies. Invest Ophthalmol Vis Sci. 2014; 55(6): 3572-3577, doi: 10.1167/iovs.14-13912, indexed in Pubmed: 24845635.

20. Cronin TH, Hertle RW, Ishikawa $H$, et al. Spectral domain optical coherence tomography for detection of foveal morphology in patients with nystagmus. J AAPOS. 2009; 13(6): 563-566, doi: 10.1016/j. jaapos.2009.09.019, indexed in Pubmed: 20006817.

21. Lee H, Proudlock FA, Gottlob I. Pediatric Optical Coherence Tomography in Clinical Practice-Recent Progress. Invest Ophthalmol Vis Sci. 2016; 57(9): OCT69-0CT79, doi: 10.1167/iovs.15-18825, indexed in Pubmed: 27409508.

22. Varsányi B, Somfai GM, Lesch B, et al. Optical coherence tomography of the macula in congenital achromatopsia. Invest Ophthalmol Vis Sci. 2007; 48(5): 2249-2253, doi: 10.1167/iovs.06-1173, indexed in Pubmed: 17460287.

23. Barthelmes D, Sutter FK, Kurz-Levin MM, et al. Quantitative analysis of OCT characteristics in patients with achromatopsia and blue-cone 
monochromatism. Invest Ophthalmol Vis Sci. 2006; 47(3): 1161-1166, doi: 10.1167/iovs.05-0783, indexed in Pubmed: 16505054.

24. Thomas MG, McLean RJ, Kohl S, et al. Early signs of longitudinal progressive cone photoreceptor degeneration in achromatopsia. Br J Ophthalmol. 2012; 96(9): 1232-1236, doi: 10.1136/bjophthalmol-2012-301737, indexed in Pubmed: 22790432.

25. Hess DB, Asrani SG, Bhide MG, et al. Macular and retinal nerve fiber layer analysis of normal and glaucomatous eyes in children using optical coherence tomography. Am J Ophthalmol. 2005; 139(3): 509-517, doi: 10.1016/j.ajo.2004.10.047, indexed in Pubmed: 15767062.

26. El-Dairi MA, Asrani SG, Enyedi LB, et al. Optical coherence tomography in the eyes of normal children. Arch Ophthalmol. 2009; 127(1): 50-58, doi: 10.1001/archophthalmol.2008.553, indexed in Pubmed: 19139338.

27. Eriksson U, Alm A. Repeatability in and interchangeability between the macular and the fast macular thickness map protocols: a study on normal eyes with Stratus optical coherence tomography. Acta Ophthalmol. 2009; 87(7): 725-730, doi: 10.1111/j.1755-3768.2008.01345.x, indexed in Pubmed: 18937816.

28. Prakalapakorn SG, Freedman SF, Lokhnygina Y, et al. Longitudinal reproducibility of optical coherence tomography measurements in children. J AAPOS. 2012; 16(6): 523-528, doi: 10.1016/j.jaapos.2012.08.011, indexed in Pubmed: 23237748.

29. Turk A, Ceylan OM, Arici C, et al. Evaluation of the nerve fiber layer and macula in the eyes of healthy children using spectral-domain optical coherence tomography. Am J Ophthalmol. 2012; 153(3): 552-559. e1, doi: 10.1016/j.ajo.2011.08.026, indexed in Pubmed: 22019223.

30. Huynh SC, Wang XY, Rochtchina $E$, et al. Distribution of macular thickness by optical coherence tomography: findings from a population-based study of 6 -year-old children. Invest Ophthalmol Vis Sci. 2006; 47(6): 2351-2357, doi: 10.1167/iovs.05-1396, indexed in Pubmed: 16723444.

31. Beharić A, Stirn-Kranjc B, Brecelj J. Elektrofiziološka ocena mrežnice otrok s prirojenim nistagmusom. Zdr Vestn. 2012; 81: 73-82.
32. Kurent A, Stirn-Kranjc B, Brecelj J. Electroretinographic characteristics in children with infantile nystagmus syndrome and early-onset retinal dystrophies. Eur J Ophthalmol. 2015; 25(1): 33-42, doi: 10.5301/ ejo.5000493, indexed in Pubmed: 25096283.

33. Eriksson U, Holmström G, Alm A, et al. A population-based study of macular thickness in full-term children assessed with Stratus OCT: normative data and repeatability. Acta Ophthalmol. 2009; 87(7): 741-745, doi: 10.1111/j.1755-3768.2008.01357.x, indexed in Pubmed: 18811639.

34. Kellner U, Wissinger B, Tippmann S, et al. Blue cone monochromatism: clinical findings in patients with mutations in the red/green opsin gene cluster. Graefes Arch Clin Exp Ophthalmol. 2004; 242(9): 729-735, doi: 10.1007/s00417-004-0921-z, indexed in Pubmed: 15069569.

35. Yang H, Yu T, Sun C, et al. Spectral-domain optical coherence tomography in patients with congenital nystagmus. Int J Ophthalmol. 2011; 4(6): 627-630, doi: 10.3980/j.issn.2222-3959.2011.06.10, indexed in Pubmed: 22553733.

36. Lee $H$, Sheth V, Bibi M, et al. Potential of handheld optical coherence tomography to determine cause of infantile nystagmus in children by using foveal morphology. Ophthalmology. 2013; 120(12): 2714-2724, doi: 10.1016/j.ophtha.2013.07.018, indexed in Pubmed: 24161406.

37. Lee H, Proudlock F, Gottlob I. Is handheld optical coherence tomography reliable in infants and young children with and without nystagmus? Invest Ophthalmol Vis Sci. 2013; 54(13): 8152-8159, doi: 10.1167/ iovs.13-13230, indexed in Pubmed: 24222299.

38. Greenberg JP, Duncker T, Woods RL, et al. Quantitative fundus autofluorescence in healthy eyes. Invest Ophthalmol Vis Sci. 2013; 54(8): 5684-5693, doi: 10.1167/iovs.13-12445, indexed in Pubmed: 23860757.

39. Giani A, Cigada M, Choudhry N, et al. Reproducibility of retinal thickness measurements on normal and pathologic eyes by different optical coherence tomography instruments. Am J Ophthalmol. 2010; 150(6): 815-824, doi: 10.1016/j.ajo.2010.06.025, indexed in Pubmed: 20965494. 